\section{TUMOR CELL-INTRINSIC STING PATHWAY IS ACTIVATED IN THE PRESENCE OF CUES FROM IMMUNE CELLS AND CONTRIBUTES TO THE ANTI-TUMOR ACTIVITY OF TUMOR CELL-TARGETED STING AGONIST ANTIBODY-DRUG CONJUGATES}

Naniye Malli Cetinbas*, Travis Monnell, Winnie Lee, Kalli Catcott, Chen-Ni Chin, Pamela Shaw, Kelly Slocum, LiuLiang Qin, Kenneth Avocetien, Keith Bentley, Susan Clardy, Brian Jones, Eugene Kelleher, Joshua Thomas, Rebecca Mosher, Dorin Toader, Jeremy Duvall, Raghida Bukhalid, Marc Damelin, Timothy Lowinger. Mersana Therapeutics, Cambridge, MA, USA

Background STING pathway agonism has emerged as a potential therapeutic mechanism to stimulate an innate anti-tumor immune response. While in principle systemic administration of a STING agonist would have many therapeutic benefits, including the delivery of STING to all tumor lesions, such an approach may be limited by toxicity. Antibody-drug conjugates (ADCs) constitute a proven therapeutic modality that is ideally suited to allow systemic administration while stimulating the innate immunity in a targeted manner. We have previously demonstrated that targeted delivery of a STING agonist with an ADC induces robust anti-tumor immune responses.

Methods Herein we investigated the mechanism of action of tumor cell-targeted STING agonist ADCs. We evaluated STING pathway activation and anti-tumor activity elicited by ADCs harboring either wild type (wt) or mutant Fc deficient in $\mathrm{Fc} \gamma$ receptor $(\mathrm{Fc} \gamma \mathrm{R})$ binding in wt or STING knockout (ko) cancer cell mono-cultures, immune cell co-cultures, and in in vivo tumor models.

Results Consistent with previous reports, the majority of cancer cell lines tested failed to induce STING pathway following STING agonist payload treatment in mono-cultures. In cancer cell:THP1 monocytic cell co-cultures, tumor-targeted STING agonist ADCs with wt Fc exhibited robust STING activation, whereas Fc-mutant ADCs or non-targeted control ADCs had minimal activity. Similar results were obtained when THP1 cells were treated in plates coated with target antigen without cancer cells, demonstrating STING activation in THP1 cells following FcrR-mediated uptake of antigenbound ADCs. Tumor-targeted Fc-wt ADCs led to marked induction of STING pathway and cancer cell-killing in cancer cell:PBMC or primary monocyte co-cultures, and complete tumor regressions in in vivo tumors. Surprisingly, while at reduced levels relative to the Fc-wt ADCs, Fc-mutant ADCs exhibited significant activity in these in vitro and in vivo models, suggesting that tumor cell-intrinsic STING pathway may be activated in the presence of cues from immune cells. Consistently, STING agonist payload treatment in the presence of conditioned media from PBMC and primary monocyte but not from THP1 cultures, led to STING activation in cancer cell mono-cultures. Moreover, Fc-mutant ADCs had diminished activity in STING ko cancer cell: PBMC or primary monocyte co-cultures, demonstrating the contribution of tumor cell-intrinsic STING activation to the anti-tumor activity elicited by tumor cell-targeted STING agonist ADCs.

Conclusions In conclusion, we demonstrated that tumor celltargeted STING agonist ADCs induce robust anti-tumor activity through mechanisms involving both Fc $\gamma \mathrm{R}$ and tumor antigen-mediated ADC internalization and subsequent induction of STING pathway in immune cells and tumor cells.

http://dx.doi.org/10.1136/jitc-2020-SITC2020.0620

\section{1 A TREGS DEPLETING, CD25-TARGETED ANTIBODY-DRUG CONJUGATE SYNERGIZES WITH TUMOR-TARGETED RADIOTHERAPY AND SYSTEMIC INTERLEUKIN-2 IN PRE-CLINICAL MODELS OF SOLID CANCERS}

Patrick van Berkel, Francesca Zammarchi*. ADC Therapeutics, London, UK

Background Regulatory $\mathrm{T}$ cells (Tregs) contribute to an immunosuppressive tumor microenvironment. High tumor infiltration by Tregs and a low ratio of Teffector cells/Tregs is often associated with poor prognosis in solid tumors. ${ }^{1}$ Tregs represent a major obstacle to cancer immunotherapies, including checkpoint inhibitors and interleukin-2 (IL-2) and are associated with tumors resistance to radiotherapy. ${ }^{2}$ CD25-ADC (a.k. a. sur301) is an antibody-drug conjugate (ADC) composed of rat monoclonal antibody PC61, directed against mouse CD25, conjugated to tesirine, a pyrrolobenzodiazepine (PBD) dimerbased protease-cleavable linker. ${ }^{3}$ Previously we showed that single low doses of CD25-ADC resulted in potent and durable antitumor activity in established syngeneic solid tumor models and the combination of a suboptimal dose was synergistic with PD-1 blockade. Tumor eradication by CD25-ADC was $\mathrm{CD} 8+\mathrm{T}$ cell-dependent and it induced protective immunity. Importantly, while CD25-ADC mediated a significant and sustained intratumoral Tregs depletion, accompanied by a concomitant increase in the number of activated and proliferating tumor-infiltrating CD8 + Teffs cells, systemic Tregs depletion was transient, alleviating concerns of potential autoimmune side effects. ${ }^{4}$

Methods Here we evaluated the anti-tumor activity of CD25ADC combined with tumor-targeted radiotherapy (RT) or systemic IL-2 in syngeneic solid tumor models.

Results To investigate the combination with radiotherapy, single low doses of CD25-ADC $(0.25$ or $0.5 \mathrm{mg} / \mathrm{kg})$ were administered intravenously either alone or in combination with image-guided focal radiation in the CT26 syngeneic model. Both doses of CD25-ADC alone induced significant anti-tumor activity compared to the vehicle control. Combination of CD25-ADC, at 0.25 or $0.5 \mathrm{mg} / \mathrm{kg}$, with focal radiotherapy resulted in synergistic anti-tumor activity with $60 \%$ and $80 \%$ tumor-free survivors (TFS), respectively, at the end of the study. Moreover, re-challenged TFS did not develop new tumors, demonstrating development of tumor-specific protective immunity.The combination of CD25-ADC and systemic IL-2 was investigated in the MC38 syngeneic model. CD25ADC was administered intravenously either alone $(0.25$ or 0.5 $\mathrm{mg} / \mathrm{kg}$, single dose) or in combination with IL-2 (0.1 or 0.8 $\mathrm{mg} / \mathrm{kg}$ ). Single doses of CD25-ADC elicited significant antitumor activity compared to the vehicle control. Combination of CD25-ADC with IL-2 resulted in enhanced anti-tumor activity in both combination groups compared to the respective single agents and the combination was synergistic at the highest IL-2 dose.

Conclusions Together, these new preclinical data show novel promising combination regimens for CD25-ADC and other commonly used anti-cancer treatments and they provide rationale for the investigation of camidanlumab tesirine (ADCT-301), a PBD-based ADC targeting human CD25, in similar clinical combinations settings.

\section{REFERENCES}

1. Shang, B., et al., Prognostic value of tumor-infiltrating FoxP3+ regulatory $T$ cells in cancers: a systematic review and meta-analysis. Sci Rep 2015;5: p. 15179.

2. Liu $S$, et al., Effects of radiation on $T$ regulatory cells in normal states and cancer: mechanisms and clinical implications. Am J Cancer Res 2015;5(11):p. 3276-85. 